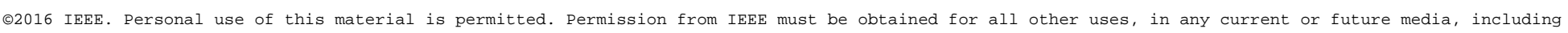

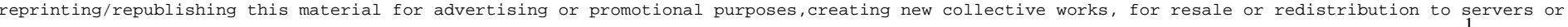
lists, or reuse of any copyrighted component of this work in other works. DOI 10.1109/TVT.2015.2468061

\title{
A Stochastic Approach for Resource Allocation with Backhaul and Energy Harvesting Constraints
}

\author{
Javier Rubio, Olga Muñoz, and Antonio Pascual-Iserte \\ Department of Signal Theory and Communications \\ Universitat Politècnica de Catalunya (UPC), Barcelona, Spain \\ Emails:\{javier.rubio.lopez, olga.munoz, antonio.pascual\}@upc.edu
}

\begin{abstract}
We propose a novel stochastic radio resource allocation strategy that achieves long-term fairness considering backhaul and air-interface capacity limitations. The base station is powered only with a finite battery that is recharged by an energy harvester. The energy harvesting is also taken into account in the proposed resource allocation strategy. The constrained scenario is often found in remote rural areas where the backhaul connection is limited and the base stations are fed with solar panels of reduced size. Our results show that the proposed scheme achieves higher fairness among the users and provides greater worst-user rate and sum-rate if an average backhaul constraint is considered.
\end{abstract}

\section{INTRODUCTION}

We consider a downlink (DL) radio resource allocation strategy for a system with limited backhaul capacity in which the base station (BS) is equipped with a finite battery recharged by an energy harvester. Although backhaul availability has been taken for granted in conventional systems, backhaul is, in general, a limited resource. This is the case of the deployment planned in the European TUCAN3G project (http://www.icttucan3g.eu). This project studies, from both the technological and socio-economical perspectives, the progressive introduction of mobile telephony and data services in isolated rural areas of developing countries. In particular, remote locations in Perú are considered. In such locations, three main challenges arise: backhaul capacity, cost of BS's, and business models adapted to people with low incomes. The solution adopted in TUCAN3G consists of an access network based on $3 \mathrm{G}$ femtocells (and its evolution to 4G) empowered by solar panels of limited size in outdoor scenarios, as well as WiLD (WiFi for Long Distances) - WiMAX - VSAT heterogeneous

Copyright (c) 2015 IEEE. Personal use of this material is permitted. However, permission to use this material for any other purposes must be obtained from the IEEE by sending a request to pubs-permissions @ ieee.org.

J. Rubio, O. Muñoz, and A. Pascual-Iserte are with the Department of Signal Theory and Communications, Universitat Politècnica de Catalunya, Barcelona, Spain e-mails: \{javier.rubio.lopez, olga.munoz, antonio.pascual\}@upc.edu

The research leading to these results has received funding from the European Commission in the framework of the FP7 Network of Excellence in Wireless COMmunications NEWCOM\# (Grant agreement no. 318306) and the project TUCAN3G (Grant agreement no. ICT-2011-601102), from the Spanish Ministry of Economy and Competitiveness (Ministerio de Economía y Competitividad) through the project TEC2011-29006-C03-02 (GRE3NLINK-MAC), project TEC2013-41315-R (DISNET), and FPI grant BES2012-052850, and from the Catalan Government (AGAUR) through the grant 2014 SGR 60. backhauling. ${ }^{1}$

The resource allocation strategy considered in this paper is developed with this scenario in mind, for which the limited capacity of the backhaul may have a huge impact on performance. The proposed strategy is described for $3 \mathrm{G}$ femtocells (based on WCDMA) as it is the solution initially considered in TUCAN3G (since it allows the use of cheap BS's and terminals). Nevertheless, the concept and methodology proposed in the paper can be extended to $4 \mathrm{G}$ femtocells (based on LTE), as will be described below in the problem formulation.

In addition to the backhaul limitation, the energy available at the BS may be a very limited resource as well. If the BS is only powered with batteries (as may happen in rural environments, for instance in the TUCAN3G deployments described above), then the battery status as well as the harvesting capabilities (if any) should also be explicitly considered in the scheduling strategy if we want to optimize the performance subject to the energy limitations. In the scenario that we will consider in this paper, the BS will be powered only by a limited battery and an energy harvesting device, e.g., solar panels that will recharge the batteries [1].

\section{A. Related Work}

With the advent of heterogeneous networks consisting of large and small cells, backhaul capacity limitations have been considered in the recent literature. For example, in [2], the authors developed a strategy to design the precoder and the power allocation in a DL scenario considering limited backhaul capacity. In [3], the authors proposed a simple scheme that performs Wyner-Ziv compress-and-forward relaying on a per-BS basis in an uplink multicell scenario where the BS's are connected to a centralized processor via rate-limited backhaul links. In [4] a strategy is developed to efficiently manage the backhaul capacity among a group of picocells. Specifically, a backhaul scheduling approach is proposed based on traffic demands along with an underlying optimum physical layer transmission scheme that maximizes the picocell utility. Sum-rate optimization with limited backhaul capacity in a

\footnotetext{
${ }^{1}$ The WiFi-LD network is already deployed and is currently in use to provide connectivity to health centers in remote areas of Perú. It will also be used to provide $3 \mathrm{G}$ connectivity (voice and data) to the general population in the area, once the access network is deployed. Such a solution meets the low energy consumption and low maintenance/installation cost constraints required in the project while allowing for an easy and progressive network upgrade as traffic demand increases.
} 
network-MIMO setup and in a coordinated multipoint (CoMP) setup was considered in [5] and [6]. A joint beamforming and clustering strategy was presented in [7]. The scenario of this work is a DL network-MMO scenario, where BS's are connected to a central processor with rate-limited backhaul links. A heuristic scheme that jointly optimizes user scheduling and power control was proposed in [8] where, cooperation among BS's via capacity-limited backhaul links was considered. Finally, a joint user association and resource allocation strategy in a multicell heterogeneous network was presented in [9], where each BS was provided with a limited backhaul capacity link.

In the previous works, the backhaul capacity limitation is introduced by imposing a maximum instantaneous aggregate traffic constraint. However, limiting the sum-rate instantaneously at each specific scheduling period to match the instantaneous backhaul rate may hamper the performance of the system in terms of the achievable long-term rates. In these circumstances, it seems less limiting to use high data rates in the access network whenever the channel conditions allow (possibly using greater instantaneous values than the average constraint imposed by the backhaul) provided that the average backhaul rate constraint is met when averaging the traffic served. That is the strategy that we follow in this paper and it is the main difference with respect to the works presented before. Note that the backhaul constraint in terms of average traffic is suitable if we assume that queues are implemented at the entrance of the access network. Note also that this relaxed constraint will increase latency on the backhaul, which we do not consider in our analysis.

\section{B. Main Contribution}

The main contribution of this paper is a fair scheduling algorithm considering a long-term backhaul constraint, the battery status of the BS, and the energy that it is being harvested. To maximize the achievable long-term rates under average traffic constraints we derive an online algorithm based on stochastic optimization tools [10]. Although not explicitly described in the paper, the conventional proportional fair (PF) is extended to incorporate instantaneous backhaul capacity constraints. Its performance is then compared with the stochastic algorithm proposed for which the average traffic served meets the average backhaul constraint, while taking advantage in an opportunistic way of the instantaneous good wireless channel conditions and also with the stochastic proposed scheme considering instantaneous backhaul constraints.

\section{Organization of the Paper}

The remainder of this paper is organized as follows. In Section II we describe the system model. Section III presents the resource allocation strategy developed in the paper. The numerical evaluation, presented in Section IV, has been carried out using models taken from remote rural locations in the forest in Perú. Finally, conclusions are drawn in Section V.

\section{SySTEM MODEL}

\section{A. System Description}

Let us consider a DL scenario composed of a single BS and several users. Because we focus on providing $3 \mathrm{G}$ connectivity, the system is based on WCDMA technology and two different types of users coexist: voice users and data users. The set of voice and data users are defined by $\mathcal{K}_{V}$ and $\mathcal{K}_{D}$, respectively, and it is assumed that voice users request a fixed service rate whereas data users request a flexible service rate.

Users in WCDMA are multiplexed using codes [11]. We assume that the network operator has already reserved a set of codes for the voice users and the remaining codes are to be allocated among the data users. Thus, the amount of available codes in each set is known and fixed at the BS.

The BS is powered only with a battery and an energy harvester. The energy harvester allows the BS to collect energy from the environment and recharge the battery (for example, solar panels). This is especially important in rural areas, where the access to the power grid may be impossible or too expensive. We consider that only causal information is available for the resource allocation strategy, i.e., only information of the past and current harvesting collections and battery dynamics will be available to execute the scheduling strategy at each particular scheduling period, yielding to an online approach.

One of the novelties of this work is that we account for a maximum backhaul rate constraint. However, instead of limiting the instantaneous access network data rates as the maximum flow allowed by the backhaul, as in [2], [3], and [9], we limit the average throughput served by the access network. That means that we allow the instantaneous rate in the access wireless links to surpass the backhaul limitation at certain time instants. This can be done whenever we have queues at the entrance of the access network and such queues are stable (which, in fact, is guaranteed by imposing that the average aggregated rate is not higher than the backhaul capacity). As we focus on fairness, in the resource allocation problem the long-term backhaul capacity is equally divided among the users with the same type of service. Accordingly, we consider in the access network resource allocation problem that the backhaul capacity is equally divided among the users with the same type of service. Fig. 1 presents the system architecture of the target rural scenario.

\section{B. Power Consumption Model and Battery Dynamics}

In this subsection, we introduce the power consumption and battery model considered in this paper. The overall power consumption at the $\mathrm{BS}$ is modeled as the addition of the radiated power, which is divided into the power devoted to pilot channels $\left(P_{\mathrm{CPICH}}\right.$ assumed to be fixed) and the power consumed by the traffic channels $\left(P_{\mathrm{BS}}(t)\right)$, and a fixed power consumed by the electronics of the BS $\left(P_{\mathrm{c}}\right)$, where $t$ denotes the scheduling period. The model considered for the last term is based on [12] and includes the power consumption of the radio frequency (RF) chains, the baseband power consumption, and the consumption of the cooling systems. The maximum 


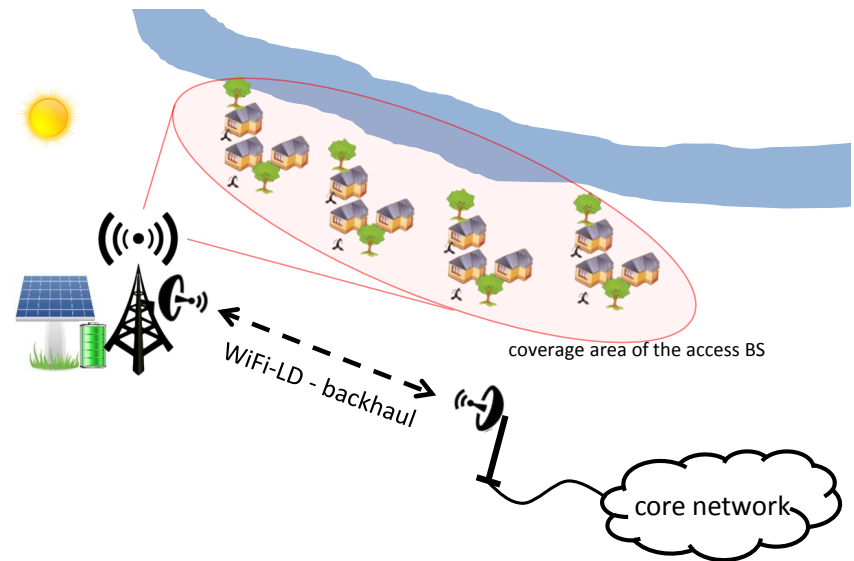

Fig. 1. Architecture of the target rural scenario under consideration in the paper. The BS is powered with a solar panel and a battery and the backhaul considered is based on WiFi-LD. The specific details of the real deployment as well as the location are explained in the simulation section.

traffic power will depend on the current battery level of the BS, as will be described in more detail later.

The overall energy consumption by the BS during the $t$-th scheduling period is

$$
E(t) \triangleq T_{s} \cdot\left(P_{\mathrm{CPICH}}+P_{\mathrm{BS}}(t)+P_{\mathrm{c}}\right), \quad \forall t,
$$

where $T_{s}$ is the duration of the scheduling period. Due to physical constraints of the amplifiers of the BS, the amount of power available for traffic services is limited, and it is denoted as $P_{\mathrm{BS}}^{\max }$, so $P_{\mathrm{BS}}(t) \leq P_{\mathrm{BS}}^{\max }$.

Let $B(t)$ be the energy stored at the battery of the BS at the beginning of the scheduling period $t$. Then at period $t+1$, the battery level is updated in general as

$$
B(t+1)=f(B(t), E(t), H(t)), \quad \forall t,
$$

where $H(t)$ is the energy harvested in Joules during the scheduling period $t$ and the function $f(\cdot): \mathbb{R}_{+} \times \mathbb{R}_{+} \times$ $\mathbb{R}_{+} \rightarrow \mathbb{R}_{+}$depends upon the battery dynamics, such as storage efficiency and memory effects. A common practice is to consider the following battery update:

$$
B(t+1)=(B(t)-E(t)+H(t))_{0}^{B^{\max }}, \quad \forall t,
$$

where $(x)_{a}^{b}$ is the projection of $x$ onto the interval $[a, b]$, i.e., $(x)_{a}^{b}=\min \{\max \{a, x\}, b\}$, which accounts for possible battery overflows and assures that the battery levels are nonnegative, and $B^{\max }$ is the battery capacity. Notice that the whole harvesting collected during period $t$ is assumed to be available in the battery at the end of the period for simplicity. In general, the total energy consumed by the BS during one period will be limited by a function of the current battery level as

$$
T_{s} \cdot\left(P_{\mathrm{CPICH}}+P_{\mathrm{BS}}(t)+P_{\mathrm{c}}\right) \leq g(B(t)), \quad \forall t,
$$

where the function $g(\cdot)$ is defined as $g(B(t)) \triangleq$ $\min \left\{T_{s}\left(P_{\mathrm{CPICH}}+P_{\mathrm{BS}}^{\max }+P_{\mathrm{c}}\right), w(B(t))\right\}$, and $w(\cdot): \mathbb{R}_{+} \rightarrow$ $\mathbb{R}_{+}$a generic continuous increasing function that satisfies $w(B(t)) \leq B(t), \forall t$. For example, if all the battery is allowed to be spent during one particular epoch, then $w(B(t))=B(t)$. Nevertheless, the approach followed in this paper is to limit the amount the battery that can be used in order to keep more energy in the battery over long periods of time. Thus, only a given fraction of the battery is allowed to be used during a particular scheduling period, i.e.,

$$
w(B(t))=\alpha \cdot B(t), \quad 0 \leq \alpha \leq 1 .
$$

\section{Energy Harvesting Model}

We assume a discretized model for the energy arrivals [13] where $H(t)$ is modeled as an ergodic Bernoulli process (which is a particular case of a Markov chain). As a result, only two values of harvested energy are possible, i.e., $H(t) \in\{0, e\}$, where $e$ is the amount of Joules contained in an energy packet. The probability of receiving an energy harvesting packet during one scheduling period depends on the actual harvesting intensity (in the case of solar energy, it depends on the particular hour of the day) and is denoted by $p(t)$. Note that a higher value of $p(t)$ will be obtained in scheduling periods where the harvesting intensity is higher, e.g., during solar presence such as during the day, and a lower value of $p(t)$ will be obtained during periods of solar absence, such as during the night.

\section{System Assumptions}

Let us collect all the channel gains, $h_{k}$, that includes the antenna gains, the path loss, and the fading, in $\mathbf{h}=\left\{h_{k}, \forall k \in\right.$ $\left.\mathcal{K}_{V} \cup \mathcal{K}_{D}\right\}$. Generally, the wireless channels depend on the specific scheduling period, $\mathbf{h}(t)$, as they vary over time but, for simplicity in the notation, we will just refer to them as $\mathbf{h}$ throughout the paper. The traffic power, $P_{\mathrm{BS}}(t)$ from (1), can be split into power for voice and data connections as $P_{\mathrm{BS}}(t)=$ $\sum_{k \in \mathcal{K}_{V}} \check{p}_{k}(\mathbf{h})+\sum_{k \in \mathcal{K}_{D}} p_{k}(\mathbf{h})$, where $\check{p}_{j}(\mathbf{h})$ and $p_{k}(\mathbf{h})$ are the instantaneous powers corresponding to the transmission toward the $j$-th and $k$-th voice and data user, respectively. Let $P_{\mathrm{RAD}}(t)=P_{\mathrm{BS}}(t)+P_{\mathrm{CPICH}}$ be the overall radiated power by the BS.

The voice users request a fixed data rate and we assume that just one WCDMA code is assigned to them. This is translated into a minimum signal to interference and noise ratio (SINR) requirement as follows:

$$
\frac{M_{V} \check{p}_{k}(\mathbf{h}) h_{k}}{\theta\left(P_{\mathrm{RAD}}(t)-\check{p}_{k}(\mathbf{h})\right) h_{k}+\sigma^{2}} \geq \Gamma, \quad \forall k \in \mathcal{K}_{V},
$$

where $M_{V}$ is the spreading factor for voice codes, $\theta$ is the orthogonality factor among DL codes [11], and $\sigma^{2}$ is the noise power. For simplicity in the notation and tractability, we will consider the following approximation ${ }^{2}$ :

$$
\theta\left(P_{\mathrm{RAD}}(t)-\check{p}_{k}(\mathbf{h})\right) h_{k}+\sigma^{2} \approx \theta P_{\mathrm{RAD}}(t) h_{k}+\sigma^{2} .
$$

On the other hand, the data users request a flexible service rate. The instantaneous throughput in the wireless access channel achieved during one particular scheduling period by the $k$-th user, $r_{k}(\mathbf{h})$, is upper bounded by the maximum

\footnotetext{
${ }^{2}$ If the number of users is relatively high, then $P_{\mathrm{RAD}}(t) \gg \check{p}_{k}$, and the approximation is fair. In any case, the approximation provides a lower bound of the actual SINR value.
} 
achievable rate that the access network is able to provide, which is formulated as

$$
r_{k}(\mathbf{h}) \leq n_{k}(\mathbf{h}) \frac{W}{M_{D}} \log _{2}\left(1+\frac{M_{D} p_{k}(\mathbf{h}) h_{k}}{n_{k}(\mathbf{h})\left(\theta P_{\mathrm{RAD}}(t) h_{k}+\sigma^{2}\right)}\right),
$$

where $M_{D}$ is the spreading factor for data codes, $W$ is the chip rate, and $n_{k}(\mathbf{h})$ is the number of codes assigned to user $k$. Notice that we have also approximated the denominator within the logarithm as in (7).

\section{Problem Formulation}

Let us introduce the following set of definitions: $\mathbf{r} \triangleq$ $\left\{r_{k}(\mathbf{h}), \forall k \in \mathcal{K}_{D}\right\}, \check{\mathbf{p}} \triangleq\left\{\check{p}_{k}(\mathbf{h}), \forall k \in \mathcal{K}_{V}\right\}, \mathbf{p} \triangleq$ $\left\{p_{k}(\mathbf{h}), \forall k \in \mathcal{K}_{D}\right\}, \mathbf{n} \triangleq\left\{n_{k},(\mathbf{h}) \forall k \in \mathcal{K}_{D}\right\}$. We formulate an optimization problem for the resource allocation strategy with backhaul and energy constraints to be executed at the beginning of each particular scheduling period, which involves finding the optimum resource allocation variables, $\mathbf{r}, \check{\mathbf{p}}, \mathbf{p}$, and $\mathbf{n}$ that maximize the minimum of the expected throughputs (note that if a scheduling criterion different from the maximin approach is to be taken, problem (9) could be extended by just reformulating the objective function accordingly):

$$
\begin{aligned}
& \underset{\mathbf{r}, \overline{\mathbf{p}}, \mathbf{p}, \mathbf{n}, P_{\mathrm{RAD}}(t)}{\operatorname{maximize}} \min _{k \in \mathcal{K}_{D}} \mathbb{E}_{\mathbf{h}}\left[r_{k}(\mathbf{h})\right] \\
& \text { subject to } \\
& C 1: \frac{M_{V} \check{p}_{k}(\mathbf{h}) h_{k}}{\theta P_{\mathrm{RAD}}(t) h_{k}+\sigma^{2}} \geq \Gamma_{k}, \quad \forall k \in \mathcal{K}_{V} \\
& C 2: \mathbb{E}_{\mathbf{h}}\left[r_{k}(\mathbf{h})\right] \leq \frac{R_{B H}-\check{R}_{B H}\left(\left|\mathcal{K}_{V}\right|\right)}{\xi\left|\mathcal{K}_{D}\right|}, \quad \forall k \in \mathcal{K}_{D} \\
& C 3: r_{k}(\mathbf{h}) \leq \frac{n_{k}(\mathbf{h}) W}{M_{D}} \log _{2}\left(1+\frac{M_{D} p_{k}(\mathbf{h}) h_{k}}{n_{k}(\mathbf{h})\left(\theta P_{\mathrm{RAD}}(t) h_{k}+\sigma^{2}\right)}\right) \\
& C 4: T_{s}\left(\sum_{k \in \mathcal{K}_{V}} \check{p}_{k}(\mathbf{h})+\sum_{k \in \mathcal{K}_{D}} p_{k}(\mathbf{h})\right) \leq \phi(B(t)) \\
& C 5: \sum_{k \in \mathcal{K}_{D}} n_{k}(\mathbf{h}) \leq N_{\max } \\
& C 6: r_{k}(\mathbf{h}) \geq 0, p_{k}(\mathbf{h}) \geq 0, n_{k}(\mathbf{h}) \geq 0, \quad \forall k \in \mathcal{K}_{D} \\
& C 7: P_{\mathrm{RAD}}=\sum_{k \in \mathcal{K}_{V}} \check{p}_{k}(\mathbf{h})+\sum_{k \in \mathcal{K}_{D}} p_{k}(\mathbf{h})
\end{aligned}
$$

where $\xi,(\xi>1)$, is an overhead considered for the data transmissions to be sent through the backhaul, $\check{R}_{B H}\left(\left|\mathcal{K}_{V}\right|\right)$ is the backhaul capacity used by the voice users ${ }^{3},\left|\mathcal{K}_{V}\right|$ being the number of voice users, $R_{B H}$ is the overall backhaul capacity, $\Gamma$ is the target SINR for the voice users, the function $\phi(\cdot)$ is related to $g(\cdot)$ in (4) as $\phi(B(t))=g(B(t))-T_{s} \cdot\left(P_{\mathrm{CPICH}}+P_{c}\right)$, and $N_{\max }$ is the number of available codes for the data users. Although all the variables in the optimization problem (9) depend on the scheduling period $t$, we only keep such explicit dependence w.r.t. time in the variable $P_{\mathrm{RAD}}(t)$ to make explicit that the temporal evolution of the battery levels has a direct

\footnotetext{
${ }^{3}$ The overall backhaul capacity required to provide voice service generally depends on the current number of voice users being served. In some cases, voice users can be jointly encoded and, thus, the overall overhead for voice users may be reduced as the number of voice users increases. Anyway, in the problem formulation and the for the sake of generality, we just use the notation $\check{R}_{B H}\left(\left|\mathcal{K}_{V}\right|\right)$.
}

impact on the maximum power to be spent for the voice and data traffic, which is not constant over time.

It is important to realize that problem (9) may not be feasible due to constraint $C 1$ as it may happen that there could not be enough power to satisfy all the target SINRs simultaneously. However, let us consider initially through the development that the problem is feasible (the feasibility condition will be developed later on). Notice that, at the optimum, $C 4$ is attained with equality; otherwise, we could re-scale all the power variables with a common positive factor higher than 1 until $C 4$ is fulfilled with equality. This would increase the objective function and all the other constraints would still be fulfilled. Because of this, we can assume that the optimum value of $P_{\mathrm{RAD}}(t)$ is $P_{\mathrm{RAD}}^{\star}(t)=\frac{\phi(B(t))}{T_{s}}+P_{\mathrm{CPICH}}$ and we can eliminate constraint $C 7$ from problem (9). Constraint $C 2$ states that the average throughput that a user is experiencing in the access network should not exceed the maximum backhaul rate assigned to this user (every user has been already assigned a portion of the backhaul, as commented above). If this is not the case, then $C 2$ could be rewritten as $\sum_{k \in \mathcal{K}_{D}} \mathbb{E}_{\mathbf{h}}\left[r_{k}(\mathbf{h})\right] \leq$ $\frac{R_{B H}-\check{R}_{B H}\left(\left|\mathcal{K}_{V}\right|\right)}{\xi}$. In any case, notice that the instantaneous (9) rates allocated to one user in the access network can be higher in some scheduling periods than the maximum backhaul peruser rate $\left(\frac{R_{B H}-\tilde{R}_{B H}\left(\left|\mathcal{K}_{V}\right|\right)}{\xi\left|\mathcal{K}_{D}\right|}\right)$ thanks to the fact that queues are considered at the entrance of the access network. The average rate constraint $C 2$ assures that the queues will be stable.

Notice that the problem is separable into voice and data users without loss of optimality. This is because the voice users do not affect explicitly the optimal value of the objective function. Thus, we can obtain the optimum power variables for the voice users (i.e., the minimum power required to satisfy constraints $C 1$ ) and, then, assign the rest of the resources to the data users. Hence, we will start by analyzing the resource allocation for the voice users in Section III-A.

Let us first provide the guidelines to reformulate the problem presented in (9) for an LTE system, which uses OFDMA as the underlying physical multiple access technology. The objective function could be expressed as it is. Constraints $C 2$, $C 4$, and $C 6$ would remain equal (in fact, in $C 6 n_{k}(\mathbf{h})$ would have to be changed by the variable representing the number of carriers). Constraint $C 1$ needs to be modified. Neither the code gain $\left(M_{V}\right)$ nor the intra-cell interference (as the access is now orthogonal) should be considered. We need to reformulate $C 3$ completely. A possible candidate for the power-rate expression would be $\sum_{j=1}^{N} \beta_{k}^{j} W \log \left(1+\frac{p_{k}^{j}(\mathbf{h}) h_{k}^{j}}{\sigma^{2}}\right)$, where $\beta_{k}^{j}$ is a new binary optimization variable that takes a value equal to 1 if carrier $j$ is assigned to user $k$ and and value equal to 0 otherwise, $p_{k}^{j}(\mathbf{h})$ represents the power per carrier, and $W$ is the carrier bandwidth. Finally, $C 5$ and $C 7$ would not be present. New constraints would have to be added: $\sum_{\forall j, \forall k} \beta_{k}^{j} \leq N$, where $N$ is number of available carriers, $\sum_{\forall k} \beta_{k}^{j}=1, \forall j$, and $\beta_{k}^{j}=\{0,1\}$. 


\section{A. Resource Allocation for Voice Users}

Voice users must satisfy a minimum SINR constraint that is related to the target data rate service:

$$
\frac{M_{V} \check{p}_{k}(\mathbf{h}) h_{k}}{\theta P_{\mathrm{RAD}}^{\star}(t) h_{k}+\sigma^{2}} \geq \Gamma, \quad \forall k \in \mathcal{K}_{V} .
$$

It is straightforward to obtain the optimum power allocation for each voice user as follows (realizing that at the optimum, constraints $C 1$ are fulfilled with equality):

$$
\check{p}_{k}^{\star}(\mathbf{h})=\frac{\Gamma\left(\theta P_{\mathrm{RAD}}^{\star}(t) h_{k}+\sigma^{2}\right)}{M_{V} h_{k}}, \quad \forall k \in \mathcal{K}_{V} .
$$

At this point, we could check the feasibility of (9). The problem is feasible if

$$
T_{s} \sum_{k \in \mathcal{K}_{V}} \check{p}_{k}^{\star}(\mathbf{h}) \leq \phi(B(t)),
$$

which could also be written only in terms of the channels of the voice users, the current battery level, and some constants as follows:

$$
\sum_{k \in \mathcal{K}_{V}} \frac{1}{h_{k}} \leq \kappa_{1} \phi(B(t))-\kappa_{2}
$$

where $\kappa_{1}=\frac{M_{V}-\left|\mathcal{K}_{V}\right| \theta \Gamma}{\sigma^{2} T_{s} \Gamma}$ and $\kappa_{2}=\frac{\left|\mathcal{K}_{V}\right| \theta P_{\mathrm{CPCH}}}{\sigma^{2}}$. If the problem is not feasible, then we should consider reducing such minimum SINR requirements (which would increase the constant term $\kappa_{1}$ ), dropping out some voice users in the scheduling period, or increasing $\phi(B(t))$ by taking a higher value for $\alpha$, but always guaranteeing that the maximum radiated constraint $P_{\mathrm{BS}}^{\max }$ is not exceeded.

\section{B. Resource Allocation for Data Users}

Now that we have considered the voice users, we can tackle the resource allocation problem for the data users by solving problem (9). Note that problem (9) is convex once we know $P_{\mathrm{BS}}^{\star}(t)$. To solve problem (9), we will reformulate it by introducing the slack variable $s$, which preserves convexity [14], as

$$
\begin{array}{ll}
\underset{s, \mathbf{r}, \mathbf{p}, \mathbf{n}}{\operatorname{maximize}} & s \\
\text { subject to } & C 2, \ldots, C 6 \text { of problem }(9) \\
& C 8: s \leq \mathbb{E}_{\mathbf{h}}\left[r_{k}(\mathbf{h})\right], \quad \forall k \in \mathcal{K}_{D} \\
& C 9: 0 \leq s \leq \frac{R_{B H}-\check{R}_{B H}\left(\left|\mathcal{K}_{V}\right|\right)}{\xi\left|\mathcal{K}_{D}\right|} .
\end{array}
$$

Notice that we have introduced an additional constraint, $C 9$. As is clear from the formulation, this constraint does not affect the optimum solution, but it will help in the numerical search of the optimum value of the new slack variable $s$. Notice also that the previous optimization problem is time-coupled (we require the future channel realizations due to the expectation operator appearing in $C 8$ ). In order to deal with such a difficult problem involving expectations, we propose to use a stochastic approximation [10]. In this approach, the constraints involving expectations are dualized, and their Lagrange multipliers are estimated stochastically at each period. Let us start by dualizing constraint $C 8$. Let $\boldsymbol{\lambda} \triangleq\left\{\lambda_{k}, \forall k \in \mathcal{K}_{D}\right\}$ be the vector of Lagrange multipliers associated with $C 8$. The partial Lagrangian is given by $\mathcal{L}_{C 8}(s, \boldsymbol{\lambda})=-s+\sum_{k \in \mathcal{K}_{D}} \lambda_{k}\left(s-\mathbb{E}_{\mathbf{h}}\left[r_{k}(\mathbf{h})\right]\right)$. In order to find the optimum $s$ we have to perform the following minimization:

$$
\underset{0 \leq s \leq \frac{R_{B H}-\widetilde{R}_{B H}\left(\left|\mathcal{K}_{V}\right|\right)}{\xi\left|\mathcal{K}_{D}\right|}}{\operatorname{minimize}} \mathcal{L}_{C 8}(s, \boldsymbol{\lambda}) .
$$

According to [10], when the objective function is linear in the optimization variable, the stochastic primal-dual algorithms present some numerical problems. This can be avoided by transforming the objective function introducing a general differentiable monotonically increasing cost function $U(\cdot)$ (e.g., the logarithm). Note that the introduction of this function does not modify the optimal value of the optimization variables (i.e., the solution is the same). Given that, setting the gradient to zero, $\nabla_{s} \mathcal{L}_{C 8}(s, \boldsymbol{\lambda})=0$ and solving yields:

$$
s^{\star}(\boldsymbol{\lambda})=\left((\dot{U})^{-1}\left(\sum_{k \in \mathcal{K}_{D}} \lambda_{k}\right)\right)_{0}^{\frac{R_{B H}-\check{R}_{B H}\left(\left|\mathcal{K}_{V}\right|\right)}{\xi\left|\mathcal{K}_{D}\right|}},
$$

where $\dot{U}(\cdot)$ is the derivative of $U(\cdot)$ and $(\dot{U})^{-1}(\cdot)$ is the inverse function of $\dot{U}(\cdot)$. Once we know the optimum $s^{\star}$, the problem (14) is updated as follows (where we have skipped in the objective function the term that does not depend on the optimization variables remaining in the optimization problem):

$$
\begin{array}{ll}
\underset{\mathbf{r}, \mathbf{p}, \mathbf{n}}{\operatorname{maximize}} & \sum_{k \in \mathcal{K}_{D}} \lambda_{k} \mathbb{E}_{\mathbf{h}}\left[r_{k}(\mathbf{h})\right] \\
\text { subject to } & C 2, \ldots, C 6 \text { of problem (9). }
\end{array}
$$

Now, we proceed to dualize constraint $C 2$. Let $\boldsymbol{\mu} \triangleq\left\{\mu_{k}, \forall k \in\right.$ $\left.\mathcal{K}_{D}\right\}$ be the vector of Lagrange multipliers associated with $C 2$. The partial Lagrangian is

$$
\begin{aligned}
\mathcal{L}_{C 2}\left(r_{k}(\mathbf{h}) ;\right. & \boldsymbol{\lambda}, \boldsymbol{\mu})= \\
& -\sum_{k \in \mathcal{K}_{D}} \lambda_{k} \mathbb{E}_{\mathbf{h}}\left[r_{k}(\mathbf{h})\right] \\
& +\sum_{k \in \mathcal{K}_{D}} \mu_{k}\left(\mathbb{E}_{\mathbf{h}}\left[r_{k}(\mathbf{h})\right]-\frac{R_{B H}-\check{R}_{B H}\left(\left|\mathcal{K}_{V}\right|\right)}{\xi\left|\mathcal{K}_{D}\right|}\right), \\
= & -\mathbb{E}_{\mathbf{h}}\left[\sum_{k \in \mathcal{K}_{D}}\left(\lambda_{k}-\mu_{k}\right) r_{k}(\mathbf{h})\right] \\
& -\sum_{k \in \mathcal{K}_{D}} \mu_{k}\left(\frac{R_{B H}-\check{R}_{B H}\left(\left|\mathcal{K}_{V}\right|\right)}{\xi\left|\mathcal{K}_{D}\right|}\right) .
\end{aligned}
$$

For given Lagrange multipliers $\lambda$ and $\boldsymbol{\mu}$, the optimization problem (14) is equivalently reformulated as (where we have skipped again in the objective function the term that does not depend on the optimization variables remaining in the optimization problem):

$$
\begin{array}{ll}
\underset{\mathbf{r}, \mathbf{p}, \mathbf{n}}{\operatorname{maximize}} & \sum_{k \in \mathcal{K}_{D}}\left(\lambda_{k}-\mu_{k}\right) r_{k}(\mathbf{h}) \\
\text { subject to } & C 3, \ldots, C 6 \text { of problem (9). }
\end{array}
$$

Notice that the expectations are no longer present in the formulation because the remaining constraints $C 3-C 6$ are applied to instantaneous resource allocation variables (without expectations) and also because the maximization of the 


\begin{tabular}{|c|}
\hline$\mu_{k}^{(q+1)}=\left(\mu_{k}^{(q)}+\epsilon\left(\mathbb{E}_{\mathbf{h}}\left[r_{k}^{\star}\left(\mathbf{h} ; \boldsymbol{\lambda}^{(q)}, \boldsymbol{\mu}^{(q)}\right)\right]-\frac{R_{B H}-\check{R}_{B H}\left(\left|\mathcal{K}_{V}\right|\right)}{\xi\left|\mathcal{K}_{D}\right|}\right)\right)_{0}^{\infty}, \forall k$ \\
\hline$\lambda_{k}(t+1)=\left(\lambda_{k}(t)+\epsilon\left(s^{\star}(\boldsymbol{\lambda}(t))-r_{k}^{\star}(\mathbf{h} ; \boldsymbol{\lambda}(t), \boldsymbol{\mu}(t))\right)\right)_{0}^{\infty}, \quad \forall k$ \\
\hline$\mu_{k}(t+1)=\left(\mu_{k}(t)+\epsilon\left(r_{k}^{\star}(\mathbf{h} ; \boldsymbol{\lambda}(t), \boldsymbol{\mu}(t))-\frac{R_{B H}-\check{R}_{B H}\left(\left|\mathcal{K}_{V}\right|\right)}{\xi\left|\mathcal{K}_{D}\right|}\right)\right)_{0}^{\infty}, \quad \forall k$ \\
\hline $\begin{array}{ll}\underset{\mathbf{p}, \mathbf{n}}{\operatorname{maximize}} & \sum_{k \in \mathcal{K}_{D}}\left(\lambda_{k}(t)-\mu_{k}(t)\right) n_{k} \frac{W}{M_{D}} \log _{2}\left(1+\frac{M_{D} p_{k} h_{k}}{n_{k}\left(\theta P_{\mathrm{RAD}}^{\star}(t) h_{k}+\sigma^{2}\right)}\right) \\
\text { subject to } & C 4, \ldots, C 6 \text { of problem (9). }\end{array}$ \\
\hline
\end{tabular}

expected value of the objective function with respect to $\mathbf{r}, \mathbf{p}$, and $\mathbf{n}$ in the current scheduling period, in this case, is the same as the maximization of the term within the expectation. The problem now resides in the computation of the optimum Lagrange multipliers which requires knowing the statistics of $r_{k}(\mathbf{h})$. If we solve the dual problem of (20), i.e., $\sup _{\boldsymbol{\lambda} \succeq 0, \boldsymbol{\mu} \succeq 0} \inf \mathcal{L}(\mathbf{r}, \mathbf{p}, \mathbf{n}, \boldsymbol{\lambda}, \boldsymbol{\mu})$, where $\succeq$ means elementwise inequality and the Lagrangian, $\mathcal{L}$, is defined in Appendix A, using a gradient approach, the optimum multipliers could be calculated recursively as shown in (21) and (22) [14], where $\epsilon$ is the step size. Note that it is not possible to compute the value of the Lagrange multipliers in real time and then solve (20), as they depend on the statistics of $r_{k}(\mathbf{h})$ that is a function not known a priori (it is the solution of the optimization problem itself). In this situation, we propose to follow a stochastic approximation [10] and eliminate this uncertainty constraint by estimating the multipliers stochastically at each scheduling period (with a noisy instantaneous unbiased estimate of the gradient) as shown in (23) and (24) (note that this philosophy is similar to the instantaneous estimation of the gradient in the classical LMS algorithm [15]).

The advantages of the stochastic techniques are threefold: $i$ ) the computational complexity of the stochastic technique is significantly lower than that of their off-line counterparts; $i i$ ) stochastic approaches can deal with non-stationarity environments; iii) the distribution of the involved random variables $\mathbf{h}$ is not required.

Once we update the values of the Lagrange multipliers, problem (20) can be solved using, for example, a primaldual approach. Notice that constraint $C 3$ can be put directly in the objective function as, at the optimum, it is fulfilled with equality, i.e., $r_{k}^{\star}(\mathbf{h} ; \boldsymbol{\lambda}(t), \boldsymbol{\mu}(t))=$ $n_{k}^{\star}(\mathbf{h} ; \boldsymbol{\lambda}(t), \boldsymbol{\mu}(t)) \frac{W}{M_{D}} \log _{2}\left(1+\frac{M_{D} p_{k}^{\star}(\mathbf{h} ; \boldsymbol{\lambda}(t), \boldsymbol{\mu}(t)) h_{k}}{n_{k}^{\star}(\mathbf{h} ; \boldsymbol{\lambda}(t), \boldsymbol{\mu}(t))\left(\theta P_{\mathrm{RAD}}^{\star}(t) h_{k}+\sigma^{2}\right)}\right)$ Thus, the resource allocation problem to be solved at the beginning of the scheduling period $t$ is the one shown in (25). Notice that we have not considered the dependency of the optimization variables with respect to the channels explicitly for the sake of simplicity in the notation. Problem (25) can be solved using, for example, a primal-dual approach, as it is described in Appendix A.
It can be shown that the sample average of the stochastic rates, $r_{k}^{\star}(\mathbf{h} ; \boldsymbol{\lambda}(t), \boldsymbol{\mu}(t))$, satisfies all the constraints in (9) and incurs minimal performance loss relative to the optimal (offline) solution of (9). This can be stated rigorously as follows: define $F(t) \triangleq \min _{k \in \mathcal{K}_{D}} \frac{1}{t} \sum_{\tau=1}^{t} r_{k}^{\star}(\mathbf{h} ; \boldsymbol{\lambda}(\tau), \boldsymbol{\mu}(\tau))$ and $f^{\star}$ as the minimum value of the objective function in (9). Then, it holds with probability one that as $t \rightarrow \infty$ : $i$ ) the solution is feasible; and $i i) F(t) \leq f^{\star}+\delta(\epsilon)$, where $\delta(\epsilon) \rightarrow 0$ as $\epsilon \rightarrow 0$. A proof of this result is not presented here due to space limitations but it can be derived following [10]. Let us introduce some important remarks here regarding problem (25):

Remark 1: the values of $\left\{\lambda_{k}\right\}$ measure how far the average rate $s^{\star}(\boldsymbol{\lambda}(t))$ is from the instantaneous rates served to the users in the access network. If the quality of the channels or the available powers are such that the instantaneous rates served in the access network are far from the target average rate $s^{\star}(\boldsymbol{\lambda}(t))$ for all the users, the sum of $\left\{\lambda_{k}\right\}$ will increase and the system will reduce the target average rate $s^{\star}(\boldsymbol{\lambda}(t))$.

Remark 2: in the access network, the values of $\left\{\lambda_{k}\right\}$ are in charge of ensuring that all average rates tend to grow simultaneously (maximin objective function). Note that, if at any temporal period $s^{\star}(\boldsymbol{\lambda}(t))>r_{k}^{\star}(\mathbf{h} ; \boldsymbol{\lambda}(t), \boldsymbol{\mu}(t))$, then $\lambda_{k}(t+1)$ grows and the priority of the $k$-th user to be served increases.

Remark 3: if at any temporal period $r_{k}^{\star}(\mathbf{h} ; \boldsymbol{\lambda}(t), \boldsymbol{\mu}(t))>$ $\frac{R_{B H}-\check{R}_{B H}\left(\left|\mathcal{K}_{V}\right|\right)}{\xi\left|\mathcal{K}_{D}\right|}$, then $\mu_{k}(t+1)>\mu_{k}(t)$. For a fixed set of $\left\{\lambda_{k}\right\}$, if $\lambda_{k}(t+1)-\mu_{k}(t+1)$ decreases, the user will have a lower priority to be served in the next period. The same reasoning could be applied if $r_{k}^{\star}(\mathbf{h} ; \boldsymbol{\lambda}(t), \boldsymbol{\mu}(t))<\frac{R_{B H}-\check{R}_{B H}\left(\left|\mathcal{K}_{V}\right|\right)}{\xi\left|\mathcal{K}_{D}\right|}$ to deal with the reverse situation.

\section{Resource Allocation Algorithm}

In this subsection, we present the overall algorithm that solves the resource allocation for the voice and data users based on the approaches presented in previous sections. This algorithm should be solved by the BS at the beginning of each scheduling period (whose duration is chosen usually according to the channel dynamics). The algorithm is summarized in Table I. Notice that in the algorithm, steps 7 to 15 correspond 
TABLE I

Algorithm for Solving Resource Allocation Problem (9)

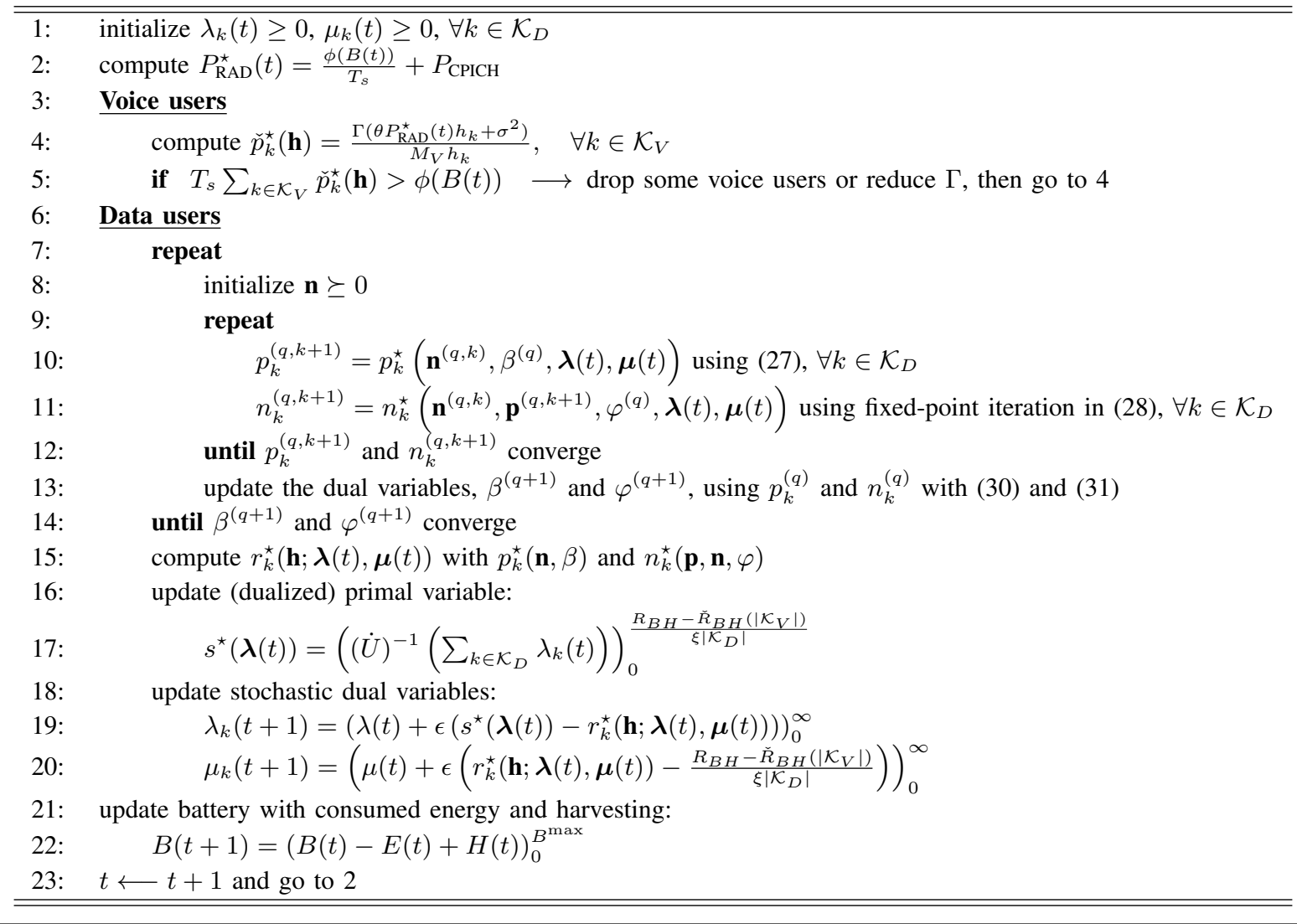

to the steps presented in Appendix A to solve the convex optimization problem (25). Note also that the computational burden of the proposed scheme is similar to the one of the conventional PF approach [16]. In steps 10 and 11, we need to solve two waterfilling-like expressions, and the rest of the steps are just simple updates.

\section{NumERICAL EvaluATION}

In this section we evaluate the performance of the proposed strategy. The scenario under consideration is composed of 1 BS, 3 voice users, and 6 data users. The maximum radiated power is $P_{\mathrm{BS}}^{\max }=9 \mathrm{dBm}$, the pilot power is $P_{\mathrm{CPICH}}=4 \mathrm{dBm}$ (which represents the $13 \%$ of the maximum radiated power, as we considered in [17]), and the fixed power is $P_{c}=3 \mathrm{dBm}$ (considering the model in [12], which was applied in [17]). The number of available codes for data transmission services is $N_{\max }=15$. All the users are mobile with a speed of 3 $\mathrm{m} / \mathrm{s}$. The instantaneous channel gain, $h_{k}$, incorporates antenna gains, Rayleigh fading with unitary power, and the path loss. The path losses correspond to a town in Perú known as San Juan (see details in [17]). The orthogonality factor is $\theta=0.35$. The code gain of data codes $M_{D}=16$ and the minimum SINR normalized with code gain for voice users is, $\frac{\Gamma}{M_{V}}=-13.7 \mathrm{~dB}$ which corresponds to a rate of $12.2 \mathrm{Kbps}$. The noise power is $\sigma^{2}=-102 \mathrm{dBm}$. The battery capacity is $B^{\max }=410 \mu \mathrm{J}$, the energy packet size is $e=30 \mu \mathrm{J}$, and $\alpha=0.3$ unless otherwise stated. The scheduling period for the data users and voice users are $2 \mathrm{~ms}$ and $20 \mathrm{~ms}$, respectively, thus, $T_{s}=2 \mathrm{~ms}$. The utility function is $U(\cdot)=\log (\cdot)$. Two backhaul capacities have been considered in the simulations: $R_{B H}=2 \mathrm{Mbps}$ and $R_{B H}=$ $500 \mathrm{Kbps}$. The amount of backhaul capacity required by the 3 voice users considered in this deployment is $\check{R}_{B H}\left(\left|\mathcal{K}_{V}\right|\right)=$ $173 \mathrm{Kbps}$. The overhead for the data transmissions is $\xi=1.2$. The step size for the update of the stochastic multipliers is $\epsilon=10^{-3}$. For a more detailed description of the simulation parameters see [17].

In the simulations, we consider as a benchmark the case where the BS is connected to the electric grid (which means equivalently that the battery remains full of energy for the whole simulation). For comparison purposes, we also show the resource allocation of the proposed strategy and the PF strategy both with an instantaneous per-user backhaul constraint $r_{k}(\mathbf{h}) \leq \frac{R_{B H}-\check{R}_{B H}\left(\left|\mathcal{K}_{V}\right|\right)}{\xi\left|\mathcal{K}_{D}\right|}$. Thanks to this, we can compare the performance of the stochastic maximin strategy with wellknown scheduling strategies and we can directly measure the improvement of the proposed stochastic scheme when we allow instantaneous access data rates to surpass the backhaul capacity. The effective length of the exponential window in the PF scheme has been set to $T_{c}=500[16]^{4}$. In the figures, PE refers to the solution of Algorithm 9, PI refers to the strategy from (9) but replacing constraint $C 2$ by an instantaneous backhaul constraint, and PF refers to the proportional fair.

\footnotetext{
${ }^{4}$ The weights of the PF scheduler are calculated as $\omega_{k}(t)=\frac{1}{T_{k}(t)}$, where $T_{k}(t)$ is the average throughput of user $k$ computed as $T_{k}(t)=$ $\left(1-\frac{1}{T_{c}}\right) T_{k}(t-1)+\frac{1}{T_{c}} r_{k}(t)$.
} 

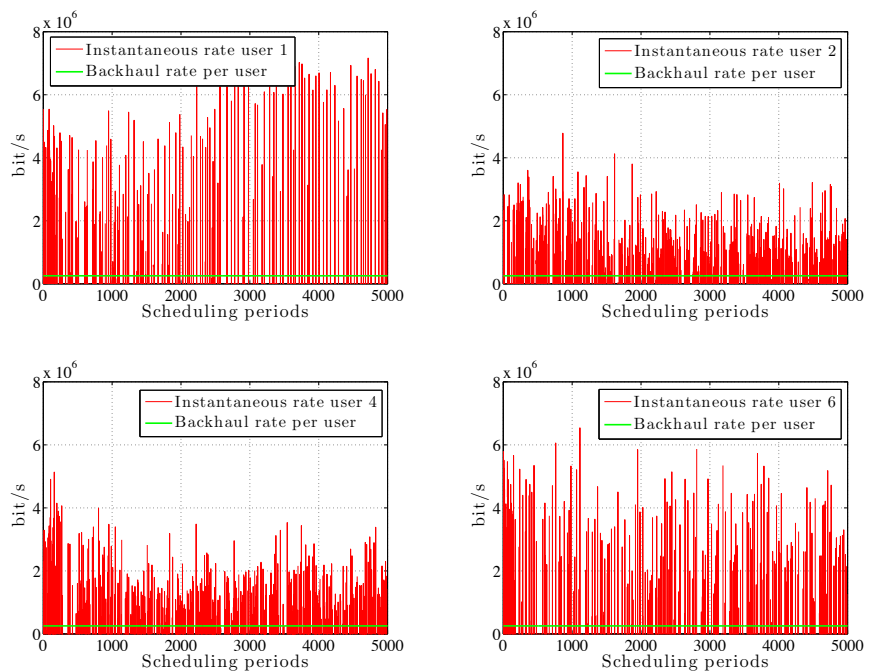

Fig. 2. Time evolution of the instantaneous data rates served at the access network and the backhaul capacity limitation per user with a backhaul capacity of $2 \mathrm{Mbps}$.

Fig. 2 presents the instantaneous data rates served at the access network of four data users out of the six. In this case, the BS is connected to the electric power grid. As we can see, the instantaneous rates are able to exceed the backhaul capacity in particular scheduling periods whereas, at the same time, the average rates fulfills the maximum backhaul capacity as it is shown in Fig. 3. Fig. 3 shows the time evolution of the expected data rates of the three approaches. At any time instant the expected rates have been estimated using $r_{k}(t)=\frac{1}{t} \sum_{\tau=1}^{t} r_{k}(\tau)$. We also plot the time evolution of $s^{\star}(\boldsymbol{\lambda}(t))$ and the per-data user backhaul rate. In this case, the $\mathrm{BS}$ is connected to the electric grid. The backhaul capacity is $R_{B H}=2 \mathrm{Mbps}$. Initially, we assume that the queues at the access network are sufficiently full so that all the bits demanded by the users are served. This allows the initial average rates to violate the backhaul capacity constraint for a short period of time (see the initial transient in the figure). This is due to the stochastic approximation of the multipliers but, in any case, when the average rates converge, they fulfill all the constraints of the original problem. As we can also see from the figure, the limitation of the rates comes from the limited resources available at the access network, i.e., the power and the codes, as the backhaul capacity is not reached. It should be also emphasized that the proposed stochastic approach provides a solution that introduces more fairness when compared with the PF approach as the average rates for the different users are quite similar. Fig. 4 depicts the same curves but now considering a backhaul capacity of $R_{B H}=500 \mathrm{Kbps}$. As we can see, now the system is limited by the backhaul and not by the limited resources of the access network. It can be appreciated in both figures the improvement of the data rates when considering average backhaul constraints instead of instantaneous backhaul constraints. Fig. 5 shows the evolution of the stochastic estimation of the Lagrange multipliers, i.e., $\lambda_{k}(t)$ and $\mu_{k}(t)$, for the cases where we have a backhaul capacity of $R_{B H}=2 \mathrm{Mbps}$ and the case of having a backhaul

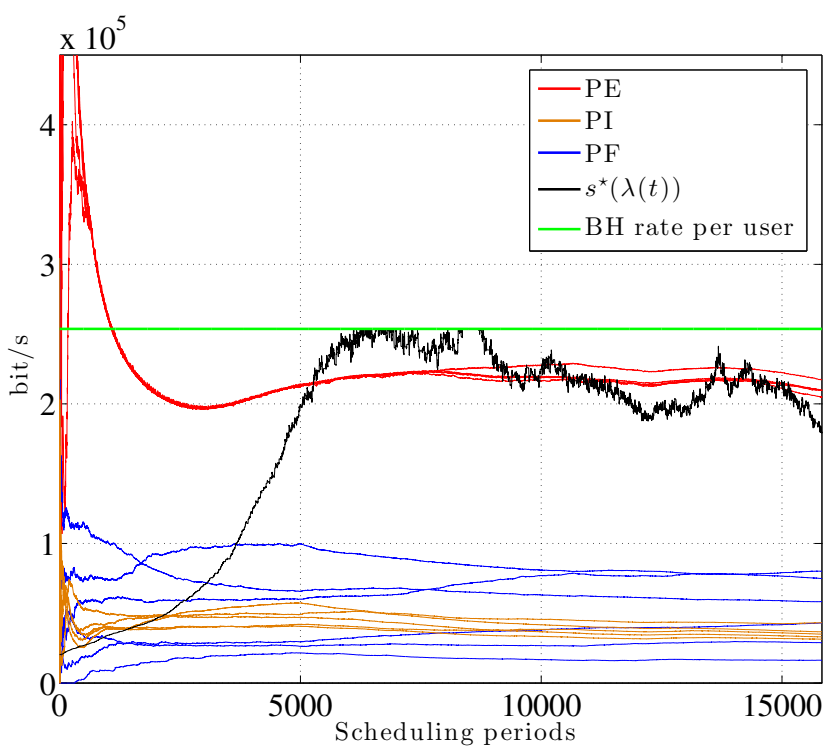

Fig. 3. Time evolution of the data rates for the different approaches and the backhaul capacity per user when the BS is connected to the electric grid with a backhaul capacity of 2 Mbps.

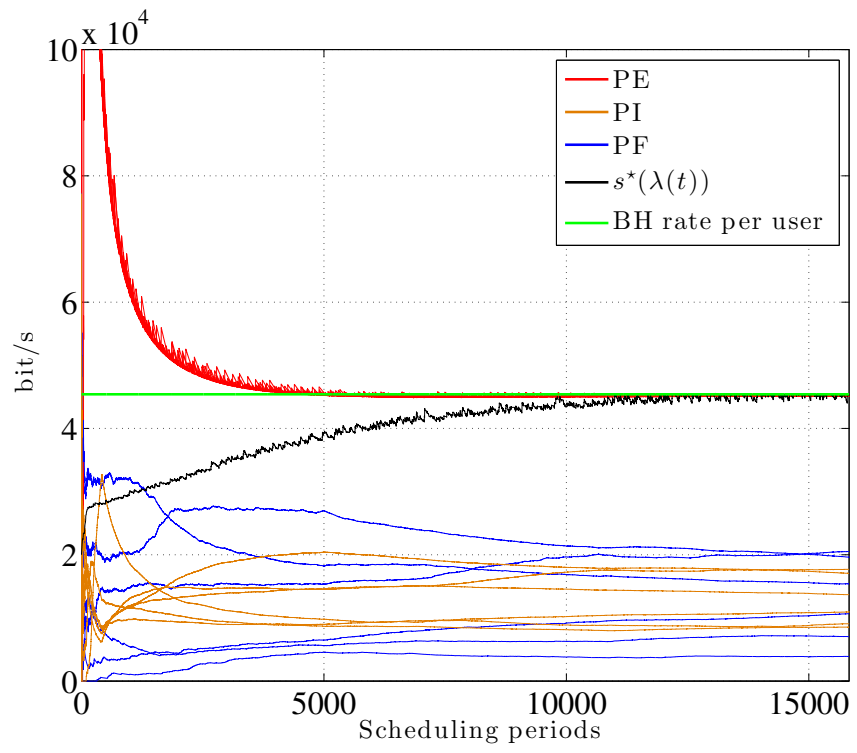

Fig. 4. Time evolution of the data rates for the different approaches and the backhaul capacity per user when the BS is connected to the electric grid with a backhaul capacity of $500 \mathrm{Kbps}$.

capacity of $R_{B H}=500 \mathrm{Kbps}$. From duality theory, we know that if the backhaul constraint is not active, i.e., if the expected rates are below the backhaul capacity, then the optimum value of the multipliers is zero. This is what we see in the figure for the case of having a backhaul capacity of $2 \mathrm{Mbps}$. On the other hand, if the system is limited by the backhaul, then the optimum Lagrange multipliers are generally not zero as the corresponding constraints become active. From the figure we see that the multipliers converge to a non-zero value. The convergence of the multipliers states that the overall stochastic approach is working properly. Fig. 6 depicts the expected rate of the worst user (shown in the left figure) and the sum of the average data rates $\left(\frac{1}{t} \sum_{\tau=1}^{t} \sum_{k \in \mathcal{K}_{D}} r_{k}(\tau)\right)$ (right figure) as a function of the overall backhaul capacity $\left(R_{B H}\right)$ 


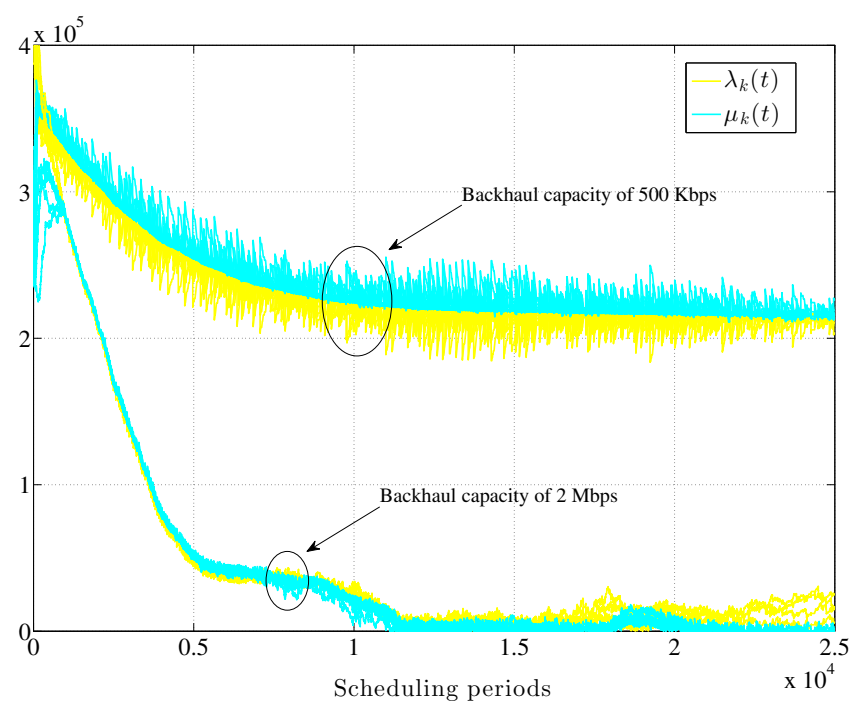

Fig. 5. Time evolution of the stochastic Lagrange multipliers for different backhaul capacities.
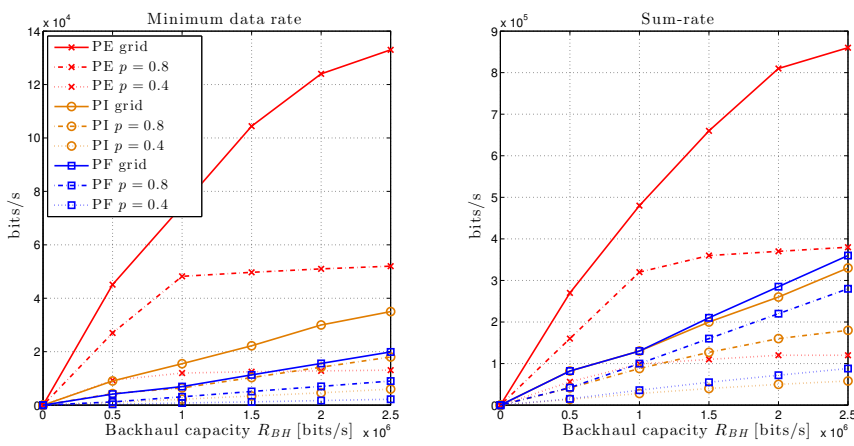

Fig. 6. Left figure: rate served in the air interface for the worst case data user versus the total backhaul capacity for different probability of energy packet $p$. Right figure: sum-rates as a function of backhaul capacity for different probability of energy packet $p$.

for the different approaches when the BS is connected to the grid and when the BS has a finite battery with different harvesting intensities $p$. Concerning the comparison between our strategy and the PF approach, we can see that the proposed scheme provides a greater data rate to the worst-user rate in all configurations and for all backhaul bandwidths. Here we emphasize again the great improvement in terms of worstrate and sum-rate that the stochastic with average backhaul constraints provides. In other words, our proposed solution achieves much more fairness as shown in Fig. 3 and Fig. 4. This is expected since we are considering explicitly the maximin criterion in the objective of our resource allocation strategy. Finally, Fig. 7 depicts the time evolution of the instantaneous battery level of the BS when the electric grid is not available for the stochastic approach. We assume that the probability of receiving an energy packet during one scheduling period is $p=0.4$ and $p=0.8$ and two values of $\alpha$ have been considered: $\alpha=0.1$ and $\alpha=1$. Recall that $\alpha=1$ means that all the battery could be used during one particular scheduling period (if the maximum power than can be radiated by the BS allows it). In can be proved that if no radiated power limitation exists at the BS and the battery never reaches its maximum value, then theoretically the expected
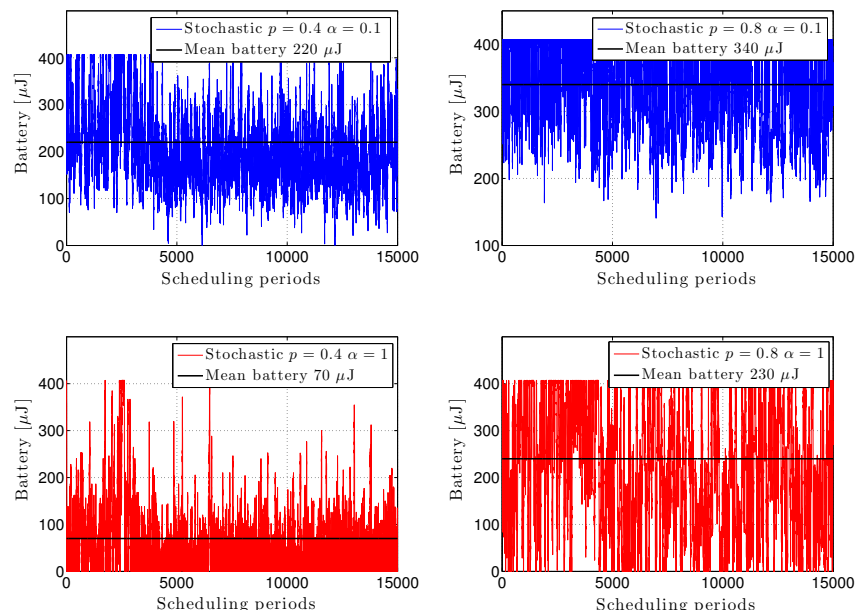

Fig. 7. Battery evolution of the proposed stochastic approach and the PF with sum constraint with a probability of energy packet $p=0.4$ and $p=0.8$ a for $\alpha=0.1$ and $\alpha=1$.

value of the battery is given by $\hat{b}=\frac{\mathbb{E}[H(t)]}{\alpha}=\frac{p \cdot e}{\alpha}$. However, due to the maximum power radiation at the BS and the battery overflows, the previous expression yields a lower bound of the true expected battery level, i.e., $\hat{b} \leq \lim _{t \rightarrow \infty} \mathbb{E}[B(t)]$. For example, if $p=0.8, \alpha=0.1$, then $\hat{b}=240 \mu \mathrm{J}$, but the figures shows $\lim _{t \rightarrow \infty} \mathbb{E}[B(t)]=340 \mu \mathrm{J}$.

\section{CONCLUSIOns}

We have proposed a resource allocation strategy based on the maximization of the minimum average data rate in a WCDMA system. By the use of stochastic optimization tools, we are able to deal with expected rates both in the objective function and in the backhaul capacity constraint, allowing the access network to offer higher rates by taking advantage of good instantaneous wireless channel conditions. We have assumed that the BS is powered with a finite battery that is able to be recharged by means of a harvesting source. The dynamics of the energy harvesting, the energy spending, and the battery have also been taken into account explicitly in the proposed resource allocation problem. Simulations results showed that the proposed approach achieves more fairness among the users when compared to the traditional PF strategy, and provides greater worst-user rate and sum-rate if an average backhaul constraint is considered instead of an instantaneous constraint.

\section{ACKNOWLEDGEMENTS}

The authors would like to thank Prof. Andrea Goldsmith for her valuable comments and suggestions that have contributed to the improvement of the quality of the paper.

\section{APPENDIX A}

In this appendix, we present the technique to solve (25) assuming that the Lagrange multipliers are known (therefore, we omit the explicit dependence of the optimization variables with respect to the stochastic Lagrange multipliers, $\boldsymbol{\lambda}(t), \boldsymbol{\mu}(t)$, for the sake of simplicity in the notation). Let $\beta$ and $\varphi$ be the Lagrange multipliers associated to constraints $C 4$ and $C 5$. There is no need to dualize constraint $C 6$ because the solution 


$$
\begin{aligned}
\mathcal{L}(\mathbf{p}, \mathbf{n} ; \beta, \varphi)= & -\sum_{k \in \mathcal{K}_{D}}\left(\lambda_{k}(t)-\mu_{k}(t)\right) n_{k} \frac{W}{M_{D}} \log _{2}\left(1+\frac{M_{D} p_{k} h_{k}}{n_{k}\left(\theta P_{\mathrm{RAD}}^{\star}(t) h_{k}+\sigma^{2}\right)}\right) \\
& +\beta\left(\sum_{k \in \mathcal{K}_{D}} p_{k}-\left(\frac{\phi(B(t))}{T_{s}}-\sum_{k \in \mathcal{K}_{V}} \check{p}_{k}\right)\right)+\varphi\left(\sum_{k \in \mathcal{K}_{D}} n_{k}-N_{\max }\right) .
\end{aligned}
$$

$$
p_{k}^{\star}(\mathbf{n} ; \beta)=\left(\frac{\left(\lambda_{k}(t)-\mu_{k}(t)\right) n_{k} W}{\ln (2) \beta M_{D}}-\frac{n_{k}\left(\theta P_{\mathrm{RAD}}^{\star}(t) h_{k}+\sigma^{2}\right)}{M_{D} h_{k}}\right)_{0}^{\infty}
$$

$$
n_{k}^{\star}(\mathbf{p}, \mathbf{n} ; \varphi)=\left(\frac{\left(\lambda_{k}(t)-\mu_{k}(t)\right) W M_{D} p_{k} h_{k}\left(\left(\theta P_{\mathrm{RAD}}^{\star}(t) h_{k}+\sigma^{2}\right) \ln (2)\right)^{-1}}{\left(\lambda_{k}(t)-\mu_{k}(t)\right) W \log \left(1+\frac{M_{D} p_{k} h_{k}}{n_{k}\left(\theta P_{\mathrm{RAD}}^{\star}(t) h_{k}+\sigma^{2}\right)}\right)-M_{D} \varphi}-\frac{M_{D} p_{k} h_{k}}{\theta P_{\mathrm{RAD}}^{\star}(t) h_{k}+\sigma^{2}}\right)_{0}^{\infty}
$$

$$
\begin{aligned}
\beta^{(q+1)} & =\left(\beta^{(q)}+\nu^{(q)}\left(\sum_{k \in \mathcal{K}_{D}} p_{k}^{(q)}-\left(\frac{\phi(B(t))}{T_{s}}-\sum_{k \in \mathcal{K}_{V}} \check{p}_{k}\right)\right)\right)_{0}^{\infty}, \\
\varphi^{(q+1)} & =\left(\varphi^{(q)}+\nu^{(q)}\left(\sum_{k \in \mathcal{K}_{D}} n_{k}^{(q)}-N_{\max }\right)\right)_{0}^{\infty},
\end{aligned}
$$

will turn out to automatically satisfy it. The Lagrangian of problem (25) is shown in (26). For given Lagrange multipliers, $\beta$ and $\varphi$, we need to minimize the Lagrangian w.r.t. the primal variables. As it will be shown next, the structure of $\mathcal{L}(\mathbf{p}, \mathbf{n}, \beta, \varphi)$ allows the minimization w.r.t. $\mathbf{p}$ and $\mathbf{n}$ to be found in closed-form. Because $\mathcal{L}(\mathbf{p}, \mathbf{n}, \beta, \varphi)$ is strictly convex and differentiable w.r.t. $\mathbf{p}$ and $\mathbf{n}$, minimization w.r.t. these variables requires to equating the corresponding partial derivatives of $\mathcal{L}(\mathbf{p}, \mathbf{n}, \beta, \varphi)$ to zero. Differentiating the Lagrangian w.r.t. the data powers, equating the derivative to zero and solving such expression for the data powers yields equation (27), where the projection on the nonnegative orthant guarantees that constraint $C 6$ is fulfilled. Proceeding similar with the optimum code allocation, if we set the partial derivative of the Lagrangian w.r.t. $n_{k}$ to zero and solve such equation for the codes, yields equation (28), where, also in this case, the projection on the nonnegative orthant guarantees that constraint $C 6$ is fulfilled. Notice that a fixed-point iteration to compute the optimum code allocation, $n_{k}^{\star}(\varphi)$, can be used in this case.

Having obtained the optimum primal variables as a function of the Lagrange multipliers, we now seek to find the optimum Lagrange multipliers to obtain the global optimum primal variables. The approach we propose to find the optimum multipliers is based on the maximization of the dual function $D(\beta, \varphi)$ [14], which is defined as the minimization of the Lagrangian w.r.t. the primal variables, i.e., $D(\beta, \varphi) \triangleq$ $\inf _{\mathbf{p}, \mathbf{n}} \mathcal{L}(\mathbf{p}, \mathbf{n}, \beta, \varphi) \equiv \mathcal{L}\left(\left\{p_{k}^{\star}(\mathbf{n}, \beta)\right\},\left\{n_{k}^{\star}(\mathbf{p}, \mathbf{n}, \varphi)\right\}, \beta, \varphi\right)$. Then, the the multipliers are obtained by solving the dual problem as

$$
\begin{array}{ll}
\underset{\beta, \varphi}{\operatorname{maximize}} & D(\beta, \varphi) \\
\text { subject to } & \beta \geq 0, \quad \varphi \geq 0 .
\end{array}
$$

Recall that the dual problem is always convex w.r.t. the dual variables and, thus, can be efficiently solved with a projected gradient method (if $D(\beta, \varphi)$ is differentiable) or a projected supergradient method if it is not differentiable [18]. A valid supergradient for each particular dual variable is given by the constraint it is associated with [18]. The update equations are shown in (30) and (31), where $q$ indicates the iteration and the step size defined as $\nu^{(q)}=\frac{Q}{\sqrt{q}}\left(\|\nabla D\|_{2}\right)^{-1}$, being $\nabla D$ the overall supergradient of the dual function, is chosen such that the diminishing conditions are fulfilled, i.e., $\lim _{k \rightarrow \infty} \nu^{(q)}=$ $0, \sum_{k \in \mathcal{K}_{D}} \nu^{(q)}=\infty$ [18]. Once we know the optimal dual variables, $\beta^{\star}$ and $\varphi^{\star}$, we can obtain the optimum power and code allocations, $p_{k}^{\star}\left(\beta^{\star}\right)$ and $n_{k}^{\star}\left(\varphi^{\star}\right)$. The proposed iterative algorithm is based on the primal-dual block coordinate descent method for the update of the primal variables $p_{k}$ and $n_{k}$ [18].

\section{REFERENCES}

[1] J. Paradiso and T. Starner, "Energy scavenging for mobile wireless electronics," IEEE Computing Pervasive, vol. 4, pp. 18-27, Jan. 2005.

[2] A. Chowdhery, W. Yu, and J. Cioffi, "Cooperative wireless multicell OFDMA network with backhaul capacity constraints," in IEEE Int'l. Conference on Comm. (ICC)), Jun. 2011.

[3] L. Zhou and W. Yu, "Uplink multicell processing with limited backhaul via per-base-station successive interference cancellation," IEEE Journal on Sel. Areas in Comm., vol. 31, pp. 1981-1993, Oct. 2013.

[4] I. Marić, B. Bostjancic, and A. Goldsmith, "Resource allocation for constrained backhaul in picocell networks," in IEEE Information Theory and Applications Workshop (ITA), Feb. 2011.

[5] H. M. Soliman et al., "Joint power and backhaul bits allocation for coordinated multi-point transmission," in International Symposium on Wireless Communication Systems (ISWCS), Nov. 2011.

[6] J. Yu et al., "Power allocation for CoMP system with backhaul limitation," in IEEE Int'l. Conference on Comm. (ICC)), Jun. 2014.

[7] B. Dai and W. Yu, "Sparse beamforming design for network MIMO system with per-base-station backhaul constraints," in IEEE International Workshop on Signal Processing Advances in Wireless Communications (SPAWC), Jun. 2014.

[8] H. Xu and P. Ren, "Joint user scheduling and power control for cell-edge performance improvement in backhaul-constrained network MIMO," in IEEE International Symposium on Personal Indoor and Mobile Radio Communications (PIMRC), Sep. 2013. 
[9] Z. Cui and R. Adve, "Joint user association and resource allocation in small cell networks with backhaul constraints," in IEEE Conf. on Information Sciences and Systems (CISS), Mar. 2014.

[10] A. Ribeiro, "Ergodic stochastic optimization algorithms for wireless communication and networking," IEEE Trans. on Signal Processing, vol. 58, pp. 6369-6386, Nov. 2010.

[11] A. Goldsmith, Wireless communications. Cambridge University Press, 2005.

[12] G. Auer et al., "How much energy is needed to run a wireless network?," IEEE Trans. on Wireless Commun., vol. 18, pp. 40-49, Oct. 2011.

[13] J. Yang, O. Ozel, and S. Ulukus, "Broadcasting with an energy harvesting rechargeable transmitter," IEEE Trans. on Wireless Communications, vol. 11, pp. 571-583, Feb. 2012.

[14] S. Boyd and L. Vandenbergue, Convex optimization. Cambridge, 2004.

[15] S. Haykin, Adaptive filter theory. Englewood Cliffs, NJ: Prentice-Hall, 2002.

[16] X. Wang, G. B. Giannakis, and A. G. Marques, "A unified approach to QoS-guaranteed scheduling for channel-adaptive wireless networks," Proceedings of IEEE, vol. 95, pp. 2410-2431, Dec. 2007.

[17] "ICT-601102 STP TUCAN3G, UMTS/HSPA network dimensioning," in Deliverable D41, available online: www.ict-tucan3g.eu, Nov. 2013.

[18] D. P. Bertsekas, Nonlinear programming. Athena Scientific, second ed., 1999. 